\title{
L'euro alimentaire en France et le partage des valeurs ajoutées
}

French "food euro" and value added distribution

\section{Philippe Boyer and Jean-Pierre Butault}

\section{(2) OpenEdition}

1 Journals

\section{Electronic version}

URL: http://journals.openedition.org/economierurale/4394

DOI: 10.4000/economierurale.4394

ISSN: 2105-2581

\section{Publisher}

Société Française d'Économie Rurale (SFER)

\section{Printed version}

Date of publication: 15 July 2014

Number of pages: $45-68$

ISSN: 0013-0559

\section{Electronic reference}

Philippe Boyer et Jean-Pierre Butault, «L'euro alimentaire en France et le partage des valeurs ajoutées », Économie rurale [En ligne], 342 I juillet-août 2014, mis en ligne le 15 juillet 2016, consulté le 20 avril 2019. URL : http://journals.openedition.org/economierurale/4394 ; DOI : 10.4000/ economierurale.4394 


\section{L'euro alimentaire en France et le partage des valeurs ajoutées}

Philippe BOYER • FranceAgriMer, Observatoire de la formation des prix et des marges des produits alimentaires, Montreuil

philippe.boyer@franceagrimer.fr

Jean-Pierre BUTAULT • INRA AgroParisTech SAE2, Nancy

butault@nancy.inra.fr

Effectuée dans le cadre de l'Observatoire de la formation des prix et des marges des produits alimentaires, l'étude consiste à décomposer le montant de la consommation alimentaire à domicile de 1995 à 2007 en importations, taxes et valeurs ajoutées des différentes branches. Les résultats font apparaître des tendances lentes, mais lourdes. La part des importations finales et en consommations intermédiaires augmente et passe de 23 à $26 \%$, et les taxes restant stables (autour de $10 \%$ ), la part des valeurs ajoutées générées dans les branches diminue $(67 \%$ en 1995 et $64 \%$ en 2007). Compte tenu des baisses des prix agricoles, la part de la valeur ajoutée de l'agriculture et de la pêche baisse de 12 à $8 \%$ entre 1995 et 2005 et la remontée des prix en 2006 et 2007 ne la porte qu'à $9 \%$. La régression de la part de la valeur ajoutée des IAA apparaît quasiment linéaire (12\% en 1995 et 10,5 \% en 2007), tout comme celle des autres industries, celle des transports restant stable. La part de la valeur ajoutée du commerce apparaît plus variable, avec un niveau équivalent en 1995 et 2007 (20\%). La part de la valeur ajoutée des services passe de 15 à $19 \%$. La composition de l'euro alimentaire évolue sous des effets " prix ", mais provient également de transformations plus profondes des modes de production et de consommation des produits alimentaires. Ces éléments ne doivent pas être oubliés dans les débats actuels sur la formation des prix alimentaires, tant sur la contractualisation entre producteurs et l'aval que sur les possibilités de développement de l'agriculture de proximité.

MOTS-CLÉS : consommation alimentaire, prix alimentaires, TES, valeur ajoutée

\section{French "food euro" and value added distribution}

Within the framework of the l'Observatoire de la formation des prix et des marges des produits alimentaires, this study consists to decompose the food consumption level into importations, taxes and value added of different sectors, in France between 1995 and 2007. The results show slow but significant tendencies. The share of importations (final products and inputs) grows from $23 \%$ to $26 \%$ over the period while the share of taxes is stable around $10 \%$. The share of value added is decreasing from $67 \%$ to $64 \%$. Considering the agriculture price decreases, the value added share of agriculture and fishing industry declines from 12\% to 8\% from 1995 to 2005 and grows to $9 \%$ thanks to the rise of prices observed in 2006 and 2007. The contribution of food industry is linearly decreasing (from 12.3\% in 1995 to 10.5\% in 2007) as it is the case of the other processing industries. The share of transportation activity remains stable. The share of the trade activities varies and attains $20 \%$ on average, the services one grows from $15 \%$ to $19 \%$. The variations of the euro-food components depend on price effects and evolutions of output processes or behavioral consumption. These elements must be put into consideration within the present debates on food price determinations, agreements between downstream activities and producers, opportunities of local agricultural developments. (JEL: M21, M41).

KEYWORDS: food consumption, food prices, input-output matrix, value added 
a question de la formation des prix à la consommation n'est pas spécifique aux produits alimentaires même si les matières agricoles connaissent d'amples fluctuations de prix qui en font un marché particulier. Pourtant, un débat récurrent persiste, en France, sur la formation des prix alimentaires, les agriculteurs accusant notamment les industries d'aval et la distribution de faire pression à la baisse sur les prix agricoles. Pour éclairer ce débat, de nombreux rapports ont ainsi été édités en France (Canivet, 2004 ; Besson, 2008 ; Lambert, 2009) et a été institué, en 2010, un Observatoire de la formation des prix et des marges des produits alimentaires (OFPM). C'est dans le cadre de cet observatoire que ce travail a été initié.

Dans la littérature économique, la question de la formation des prix alimentaires est abordée de plusieurs points de vue :

- À travers une observation de l'évolution des prix, et des coûts et bénéfices unitaires à différents stades. Les travaux de l'OFPM visent ainsi à décomposer les prix au détail de différents produits alimentaires en valeur de la matière première agricole incorporée et « marges brutes » de transformation (ou valeurs ajoutées à la matière première) et de commercialisation (ou marges commerciales proprement dites), puis en coûts de production (ou de distribution) et résultats nets, dans les entreprises de la filière (https://observatoire-prixmarges. franceagrimer.fr).

- Dans des études économétriques sur la transmission des prix entre production et consommation (cf. par exemple, pour la France, Hassan, Simioni, 2004 ; Rouchet, 2002 ; les études étant plus nombreuses dans les pays en développement).

- Dans des approches en termes d'économie industrielle ou d'économie politique visant à apprécier les pouvoirs de marché (Alain et al., 2003 ; Chambolle et al., 2007 ; Chantrel et al., 2009).
- Dans des approches plus macroéconomiques, s'appuyant notamment sur les tableaux entrées-sorties, pour cerner les relations entre les secteurs (Butault, 2008) ou appréhender la notion de complexe agroalimentaire (Rastoin, Ghersi, 2010).

C'est dans cette dernière perspective que se situe cette étude. La démarche suivie s'inspire en fait des travaux de l'United states departement of agriculture (USDA) - (http://www.ers.usda.gov/Data/ FoodDollar/) sur le «food dollar», séries annuelles présentant une décomposition de la demande alimentaire à différents niveaux, à partir des tableaux entrées-sorties (TES) américains (Canning, 2011). La méthode de l'USDA s'appuie sur une relation fondamentale, issue des travaux de Leontief $(1966,1986)$, entre les valeurs ajoutées des branches et la demande finale. Elle a déjà été utilisée dans de nombreux travaux américains sur la consommation alimentaire (voir notamment Schluter et al., 1998). Comme tout exercice de ce type, la principale limite tient à l'hypothèse de coefficients fixes de production, hypothèse que l'on retrouve à différents stades de la décomposition.

Si la démarche est spécifique aux produits alimentaires, elle rejoint l'objet de préoccupations et de travaux plus généraux sur le partage de la valeur ajoutée (Cotis, 2009).

Les TES publiés par Eurostat, sur la France, pour 1995, 1997 et de 1999 à 2007 constituent la principale base de données utilisée, avec les équilibres ressourcesemplois de l'INSEE. Compte tenu de ces données, la décomposition ne porte que sur la dépense finale des ménages en produits alimentaires (hors tabac) et n'intègre pas, contrairement aux travaux de l'USDA, la restauration et la livraison de repas à domicile (les food services). Cette analyse conduit essentiellement à cerner ce qui, sur 100 euros de dépenses alimentaires, revient aux importations, aux taxes et aux valeurs ajoutées des différentes branches. 
RECHERCHES

Philippe BOYER, Jean-Pierre BUTAULT

\section{Méthode}

\section{La relation entre demande finale et production}

Les tableaux entrées-sorties s'appuient sur la relation comptable d'égalité entre les ressources et les emplois en biens et services d'un pays :

$$
P+X=C^{I}+C^{F}+E+F
$$

$P$ étant la production, $X$ les importations, $C^{I}$ les consommations intermédiaires, $C^{F}$ la consommation finale, $E$ les exportations et $F$ la formation brute du capital fixe et la variation des stocks.

Supposons provisoirement une économie sans importation : en agrégeant les éléments de la demande finale $D$, cette relation s'écrit de manière simplifiée :

$$
P=C^{I}+D
$$

Avec plusieurs branches dans l'économie, l'égalité (2) s'écrit de façon matricielle :

$$
[D]=[P]-\left[C^{I}\right]
$$

$[D],[P]$ et $\left[C^{I}\right]$ étant les vecteurs colonnes de la demande finale, de la production et des consommations intermédiaires des branches $1, \ldots \mathrm{i}, \ldots, \mathrm{n}$.

$\mathrm{Si}$ la technologie est à coefficients fixes $\left(a_{i j}=\frac{C_{i}^{I, j}}{P_{j}}\right)$ et $[A]$ la matrice carrée de ces coefficients techniques, on a :

$$
\left[C^{I}\right]=[A] \cdot[P]
$$

et on retrouve les relations bien connues entre production et demande finale :

$$
\begin{aligned}
& {[D]=[P] \cdot[1-A]} \\
& {[P]=[1-A]^{-1} \cdot[D]}
\end{aligned}
$$

Cette dernière relation, utilisée dans tout modèle de type Leontief, permet d'estimer la production finale qu'il faut réaliser dans les différentes branches pour satisfaire un niveau donné.

\section{La relation entre valeurs ajoutées et demande finale}

Une relation moins connue - mais fondamentale pour notre objectif - est celle qui relie les valeurs ajoutées et les demandes finales (Stoléru, 1967). Au niveau macroéconomique (toujours dans une économie sans importations), cette relation est évidente : la valeur ajoutée est par définition égale à la production moins les consommations intermédiaires, et cette différence est bien la demande finale. Donc, si on note $V A, C^{I}$ et $P$, respectivement la valeur ajoutée, les consommations intermédiaires et la production toutes branches confondues :

$$
P-C^{I}=V_{A} \text { et } P-C^{I}=D,
$$

donc $V_{A}=D$

Dans l'hypothèse de technologie à coefficients techniques $a_{i j}$ fixes entre consommations intermédiaires $i$ et produits $j$, on a une relation simple entre valeur ajoutée et production de chaque branche $j$ :

$$
V A_{j}=P_{j}\left(1-\sum_{i=1}^{n} a_{i j}\right)
$$

avec $\left(1-\sum_{i=1}^{n} a_{i j}\right)$, rapport entre la valeur ajoutée de la branche $j$ et sa production, ou taux de valeur ajoutée, qu' on notera ciaprès $v_{j}$.

Soit, sous forme matricielle :

$$
[V A]=\left[V^{\prime}\right][P]
$$

$\left[V^{\prime}\right]$ étant la matrice carrée à $\mathrm{n}$ lignes et colonnes dont la diagonale est constituée par les taux de valeur ajoutés $v_{j}$, ses autres valeurs étant nulles.

Des égalités (5) et (7), on en tire :

$$
[V A]=\left[V^{\prime}\right][1-A]^{-1}[D]
$$

D'où la matrice de coefficients $[W]$ :

$$
[W]=\left[V^{\prime}\right][1-A]^{-1}
$$

La matrice $[W]$ est une matrice carrée dont les coefficients $\left(w_{i j}\right)$ donnent, en 
colonne, la composition de la demande finale d'une branche $j$ en valeur ajoutée de toutes les branches $i^{1}$.

\section{Introduction des importations dans la relation entre valeurs ajoutées et demandes finales}

Les importations constituent des biens et services qui sont, par nature, produits par des facteurs de production extérieurs au pays considéré. Leur introduction modifie donc la relation entre les valeurs ajoutées et les demandes finales.

Les importations $X$ sont, soit utilisées en consommations intermédiaires $\left(X_{C I}=\right.$ $C_{X}^{I}$ ), soit constituent une demande finale $\left(X_{D}=D_{X}\right)$. La relation d'équilibre entre ressources et emplois s'écrit alors :

$P+\left(X_{C I}+X_{D}\right)=\left(C_{I N T}^{I}+C_{X}^{I}\right)+D_{I N T}+D_{X}$

$C_{I N T}^{I}$ et $D_{I N T}$ étant les consommations intermédiaires et la demande finale de biens et services d'origine domestique. En retirant des deux côtés de l'expression précédente $X_{D}$ et $D_{X}$ on obtient :

$$
P-\left(C_{I N T}^{I}+C_{X}^{I}\right)+X_{C I}=D_{I N T}
$$

puis en soustrayant les consommations intermédiaires de la production, on obtient :

$$
V A+X_{C I}=D_{I N T}
$$

Soit, en notant $V A_{I N T}$ le premier terme, que l'on désignera par « valeur ajoutée d'origine domestique (ou intérieure) » :

$$
V A_{I N T}=D_{I N T}
$$

\footnotetext{
1. On a, par exemple, la part de la demande finale des Industries agroalimentaires (IAA) qui revient en valeur ajoutée à l'agriculture. On peut aussi décomposer la valeur ajoutée de toute branche i en demandes finales de toutes les branches $\mathrm{j}$ et obtenir par exemple les parts respectives de la valeur ajoutée de l'agriculture générées par la demande finale des IAA et des autres branches : résultats présentés dans Butault, Boyer (2012).
}

On peut ainsi retrouver tous les éléments du calcul précédent :

- la relation fondamentale relie demandes finales et valeurs ajoutées d'origine domestique ;

- la matrice des coefficients techniques et les taux de valeur ajoutée ne portent que sur la production intérieure et les consommations intermédiaires d'origine domestique.

Les importations de produits finaux sont une composante de la demande finale et les importations de consommations intermédiaires sont une utilisation de la valeur ajoutée d'origine domestique, au même titre que les salaires et l'excédent brut d'exploitation (EBE) des branches (avant taxes et subvention d'exploitation).

\section{Les sources}

L'étude s'appuie sur les TES publiés par Eurostat, sur la France, pour 1995, 1997 et entre 1999 et $2007^{2}$. La décomposition de la consommation porte sur les branches de l'agriculture, de la pêche et des IAA (sans tabac). Pour ce faire, on est revenu, pour ces branches, aux équilibres ressources-emplois établis par l'INSEE pour construire les TES. Ces équilibres donnent notamment une répartition des marges de commerce et de transport entre consommations intermédiaires, consommation finale et exportations. Ils sont donc utiles pour isoler ces marges sur la consommation finale.

2. Ceux-ci sont issus des TES français, établis dans la base 2000 (Braibant, 2008, 2011). Les tableaux 2008 et 2009 sont disponibles, mais établis en base 2005, avec une nomenclature différente des précédents, dans laquelle le tabac n'est plus dissocié des produits des industries alimentaires, ce qui nécessite des corrections approximatives si l'on veut raccorder ces années aux précédentes. C'est la raison pour laquelle on a préféré se centrer sur l'analyse de 1995-2007 pour s'en tenir à une base homogène. 


\section{Les TES d'Eurostat}

Les TES d'Eurostat font l'objet d'un manuel (Eurostat, 2008) et la construction des TES sur la France est décrite dans une note de l'INSEE (Braibant, 2011). Ils se composent de cinq tableaux.

Les deux premiers tableaux décrivent les équilibres entre les ressources et les emplois dans une présentation équivalente à celle des TES français. Ces équilibres partent de la production $\left(P_{P B}\right)$ exprimée au prix de base (cf. encadré 1), les emplois étant chiffrés aux prix d'acquisition (Braibant, 2008). Pour retrouver l'équilibre entre ressources et emplois, sont ajoutées, à la production $P_{P B}$ et aux importations $X$, les marges de commerce $M_{C}$, les marges de transport $M_{T}$ et le solde des taxes et des subventions $T \_S$. En notant $E$ et $F$ respectivement, les exportations et la formation brute de capital fixe, la relation d'équilibre entre ressources et emplois est :

$$
\begin{gathered}
P_{P B}+X+M_{C}+M_{T}+T_{-} S= \\
C^{I}+C^{F}+E+F
\end{gathered}
$$

Plusieurs contraintes spécifiques des TES français empêchent de les utiliser pour appliquer la méthode décrite plus haut. Ainsi, compte tenu du traitement particulier du commerce et des transports dans le TES français, il n'est pas possible de construire une matrice carrée des coefficients techniques : les branches de commerce et de transport ne figurent pas dans le tableau des échanges interindustriels et donc n'apparaissent pas dans les consommations intermédiaires. Par ailleurs, les importations ne sont pas ventilées selon leur utilisation en consommations intermédiaires ou finales.

Les trois autres tableaux des TES d'Eurostat sont chiffrés aux prix de base et distinguent l'ensemble de l'économie, l'économie intérieure et les importations.

Le commerce et le transport sont traités comme les autres branches. La matrice des
Encadré 1. Comptes aux prix de production, aux prix de base et aux prix d'acquisition

Les TES peuvent être établis aux prix de production, aux prix de base et aux prix d'acquisition :

- Les prix de production correspondent aux prix perçus par les entreprises avant taxes et subventions sur les produits.

- Les prix de base incluent les taxes et les subventions à la production.

- Les prix d'acquisition correspondent aux prix d'achat des produits par les utilisateurs, taxes, subventions, marge de commerce et marges de transport incluses.

coefficients techniques est donc carrée. La distinction des importations entre produits finaux et consommations intermédiaires est effectuée. La matière première du travail présenté dans cet article est donc l'utilisation du TES pour la production intérieure qui reprend dans une ligne les importations pour les consommations intermédiaires et pour la consommation finale. Mais, dans ces tableaux, le commerce et le transport font l'objet d'une demande de produits finals et la consommation alimentaire n'est pas chiffrée aux prix d'acquisition. Une étape supplémentaire est nécessaire pour effectuer ce passage.

\section{Deux corrections apportées aux TES d'Eurostat}

Le TES de la France version Eurostat a été établi, à partir du TES français, selon des règles conformes à la comptabilité nationale européenne (Braibant, 2011). Certaines de ces règles prennent mal en compte certaines spécificités de la branche agricole. Deux corrections ont ainsi été apportées à ce TES.

- L'inscription de la production de vin en production de la branche agricole et en production et consommation intermédiaire de la branche IAA. 
La construction de TES se heurte toujours à une difficulté s'agissant du traitement de la production de vin.

Dans le TES d'Eurostat aux prix de base, la production de vin, incluant la viticulture et la vinification, est considérée comme relevant de la branche des IAA, à laquelle sont affectées les consommations intermédiaires afférentes, estimées sur la base de la structure en consommations intermédiaires de la branche agricole (Braibant, 2011). Cette approche d'Eurostat diminue la valeur ajoutée de la branche agricole et augmente celle des IAA.

Pour revenir à la notion française de la valeur ajoutée de la branche agricole, incluant la production de vin, on a tout d'abord réaffecté cette production et ses consommations intermédiaires à l'agriculture.

Ensuite, la consommation finale de vin étant, dans le TES, incluse dans celle des produits des IAA, une deuxième correction a dû être introduite. Pour conserver l'équilibre entre ressources et emplois, on a ainsi considéré que le vin constituait par ailleurs une consommation intermédiaire des IAA achetés à l'agriculture et donc aussi une production des IAA. Par rapport au TES initial, la valeur ajoutée entre l'agriculture et les IAA est rééquilibrée. Au niveau de la France, la valeur ajoutée ne change pas : la seule modification concerne une augmentation de la production - la production de vin étant comptée deux fois - (en agriculture et dans les IAA), qui est compensée par une augmentation d'un même montant des consommations intermédiaires.

- L'élimination des aides directes aux produits agricoles du prix de base et leur inscription en subventions d'exploitation. La consommation finale que l'on cherche à décomposer est exprimée en prix d'acquisition, c'est à dire en prix de production augmentés des taxes sur les produits et des marges de commerce et de transport supportées in fine par le consommateur. S'agissant de la production agricole, les données disponibles doivent être retraitées pour être exprimées en prix de production, n'incorporant pas les aides directes aux produits, acquittées par le contribuable, mais pas par le consommateur. La valeur ajoutée induite par la consommation alimentaire doit être ainsi évaluée hors aides directes aux produits.

Rappelons que pour compenser une baisse des prix agricoles induite par un démantèlement des interventions sur le marché, la réforme de la PAC de 1992 a instauré un système d'aides directes aux exploitations (Butault, 2004). Ces aides ont été versées en fonction des surfaces et des têtes de bétail, sans lien direct avec le niveau de production. La réforme de 1999 a uniformisé l'aide par hectare pour toutes les grandes cultures. Eurostat et l'INSEE ont considéré ces aides comme des aides aux produits et les ont donc intégrées au prix de base. Ce choix a été contestable dans la mesure où ces subventions ne dépendaient pas directement des niveaux de production. Après 1999, Eurostat et l'INSEE ont affecté les subventions des grandes cultures aux produits, par simple règle de trois, au prorata des surfaces. Dans les faits, aucun produit n'était subventionné individuellement. Avec la réforme de 2003, ces aides ont été en partie « découplées » à partir de 2006 (Butault, 2007) et d'un point de vue statistique, selon l'INSEE et Eurostat, elles ont été considérées comme subventions d'exploitation. Au niveau de la branche agricole, les aides aux produits, au sens d'Eurostat et de l'INSEE, sont ainsi passées de 7,6 milliards d'euros à 2,5 milliards d'euros entre 2005 et 2006, alors que les subventions d'exploitation passaient de 2,2 à 7,5 milliards. Dans la mesure où le classement des subventions issues de la réforme de la PAC de 1992 comme aides aux produits est contestable, et pour 
éviter des « sauts » entre 2005 et 2006, une correction a été effectuée en considérant les aides aux produits comme des subventions d'exploitation.

Ceci a évidemment pour conséquence de diminuer la valeur ajoutée brute de l'agriculture, par exemple en 2005, de 7,5 milliards d'euros (23,5 milliards d'euros contre 31 milliards d'euros) sans affecter l'excédent brut d'exploitation (24,1 milliards d'euros).

Il aurait sans doute été préférable de partir, comme dans la méthode américaine, de tableaux chiffrés aux prix de production, pour toutes les branches. Les données disponibles ne le permettaient pas. Le prix de base inclut des taxes et des subventions qu'on ne peut pas identifier exactement et que l'on retrouve en utilisation de la valeur ajoutée des branches ${ }^{3}$. Il en résulte une petite imprécision sur le montant des taxes et subventions, avec une très légère incidence sur la valeur ajoutée des branches.

\section{Les étapes du travail}

Le point de départ est le TES au prix de base (cf. annexe 2), pour la production et la demande finale domestiques. Les tableaux d'Eurostat distinguent 59 branches. Les branches ont été réagrégées et on a retenu un découpage en 12 branches. Les branches contribuant à la consommation alimentaire à domicile sont l'agriculture, la pêche, les industries agroalimentaires (IAA) et l'industrie du tabac. Ces branches ne produisent pas seulement pour la consommation alimentaire à domicile : les produits agricoles sont utilisés dans la restauration collective, mais aussi à des fins non alimentaires comme, par exemple le textile, le bâtiment, la chimie ou encore l'énergie... La restauration collective

3. En tant que différence, pour les consommations intermédiaires, entre le prix de base et le prix d'acquisition. n'est pas séparée, dans la nomenclature, de l'hôtellerie. Il n'est donc pas possible, comme dans la méthode américaine, de décomposer la consommation alimentaire à l'extérieur et la dépense en service de repas livrés à domicile. Au stade final, le tabac n'a pas été retenu dans la consommation alimentaire, compte tenu du poids des taxes (12 milliards d'euros en 2005). Le commerce et le transport sont traités comme les autres branches et présentent notamment une demande finale. La démarche comporte plusieurs étapes :

- Par la méthode présentée, les demandes finales des produits domestiques (au prix de base), dont celles des produits alimentaires, sont décomposées en valeurs ajoutées intérieures des différentes branches. Ces valeurs ajoutées sont réparties entre les salaires, l'EBE des branches (avant taxes et subvention d'exploitation), les importations en consommations intermédiaires et les taxes en supposant une structure unique par branche.

- Pour les produits alimentaires, ces structures sont appliquées à la consommation finale toujours selon l'hypothèse de coefficients fixes pour la demande finale d'une branche, quels que soient ses emplois (consommation finale, exportations et formation brute de capital fixe $-\mathrm{FBCF}$ ).

- Jusque-là, la consommation alimentaire est mesurée aux prix de base et ne concerne que les produits intérieurs. Il faut y ajouter les produits importés et passer aux prix d'acquisition, en prenant en compte notamment les marges commerciales et de transport. Celles-ci sont données, pour la consommation finale, dans les équilibres ressourcesemplois de l'INSEE. Les marges sont la production du commerce et du transport et font l'objet d'une demande finale : leur décomposition en valeurs ajoutées est effectuée dans la première étape. Il faut donc ajouter celles-ci aux valeurs 
ajoutées précédemment calculées, au prix de base, pour la consommation alimentaire. Ce calcul s'appuie toujours sur une hypothèse forte, de type Leontief. Les taux de marges sont spécifiques à la consommation alimentaire, mais un euro de marge sur les produits alimentaires a la même décomposition en valeurs ajoutées qu'un euro de marge sur les autres produits.

- On boucle en calculant les taxes sur la consommation par solde entre la consommation aux prix d'acquisition et la consommation avec marges. Les équilibres ressources-emplois de l'INSEE permettent de vérifier que le bouclage est effectif.

\section{Le calcul du contenu en emplois} de la consommation alimentaire

Les TES européens fournissent, par branche, l'emploi mesuré par le nombre de personnes employées ${ }^{4}$. D'autres indicateurs auraient été plus pertinents, notamment le nombre d'équivalents temps plein. Par branche, on peut ainsi calculer un rapport entre emplois et valeur ajoutée (salaires + EBE). Nous avons ensuite appliqué ces rapports aux valeurs ajoutées générées par la consommation alimentaire, pour estimer son contenu en emplois.

\section{Résultats}

Vision d'ensemble de la décomposition des dépenses alimentaires pour la période 1995-2007

Dans une première approche synthétique, le graphique 1 donne les résultats moyens de la décomposition des dépenses alimentaires pour la période 1995-2007.

4. Le capital fixe est aussi donné, mais pas pour toutes les années. Cette donnée peut être utilisée, comme celle sur l'emploi, afin de calculer le capital fixe induit par la demande alimentaire (Butault, Boyer, 2012).
Entre 1995 et 2007, le montant annuel de la consommation alimentaire est de 142 milliards d'euros courants (23 milliards de produits agricoles, 3 milliards de produits de la pêche et 116 milliards de produits des IAA).

- La part des importations atteint presque le quart de ce montant $(24,4 \%)$ qui se partage presque en part égale entre les produits alimentaires finals $(12,7 \%)$ et les biens importés utilisés en consommations intermédiaires $(11,7 \%)$.

- La valeur ajoutée des branches représente $65,8 \%$ du montant de la consommation alimentaire, dont $34,2 \%$ en salaires et 31,6\% en EBE des entreprises avant subvention d'exploitation.

- La part de la valeur ajoutée de l'agriculture et de la pêche n'est que $10 \%$ et celle des IAA de $11,5 \%$.

- Ces parts s'élèvent par contre à 20,3\% pour le commerce et $17,6 \%$ pour les services.

- La valeur ajoutée des autres industries et du transport ne concourt que pour $4,2 \%$ et 2,4\% du montant de l'euro alimentaire.

- Les taxes représentent $10 \%$ des dépenses alimentaires. Sur la période étudiée, la plupart des produits alimentaires ne sont taxés qu'à un taux réduit de TVA $(5,5 \%)$, mais le taux normal de 19,6\% s'applique notamment aux boissons alcoolisées qui font, par ailleurs, l'objet de taxes spécifiques.

\section{L'évolution des volumes}

et des prix réels entre 1995 et 2007

L'objectif de l'étude n'est pas de cerner les effets respectifs de l'évolution des prix et des volumes sur la formation des revenus (à la différence d'autres travaux ; cf. Butault, 2008), mais, afin d'expliquer les évolutions de certaines composantes de « l'euro alimentaire », il est nécessaire de retracer l'évolution des volumes et des prix réels (déflatés par l'indice du prix du PIB) 
RECHERCHES

Graphique 1. Décomposition de $100 €$ de dépense alimentaire en valeurs ajoutées, importations et taxes (résultats moyens 1995-2007)

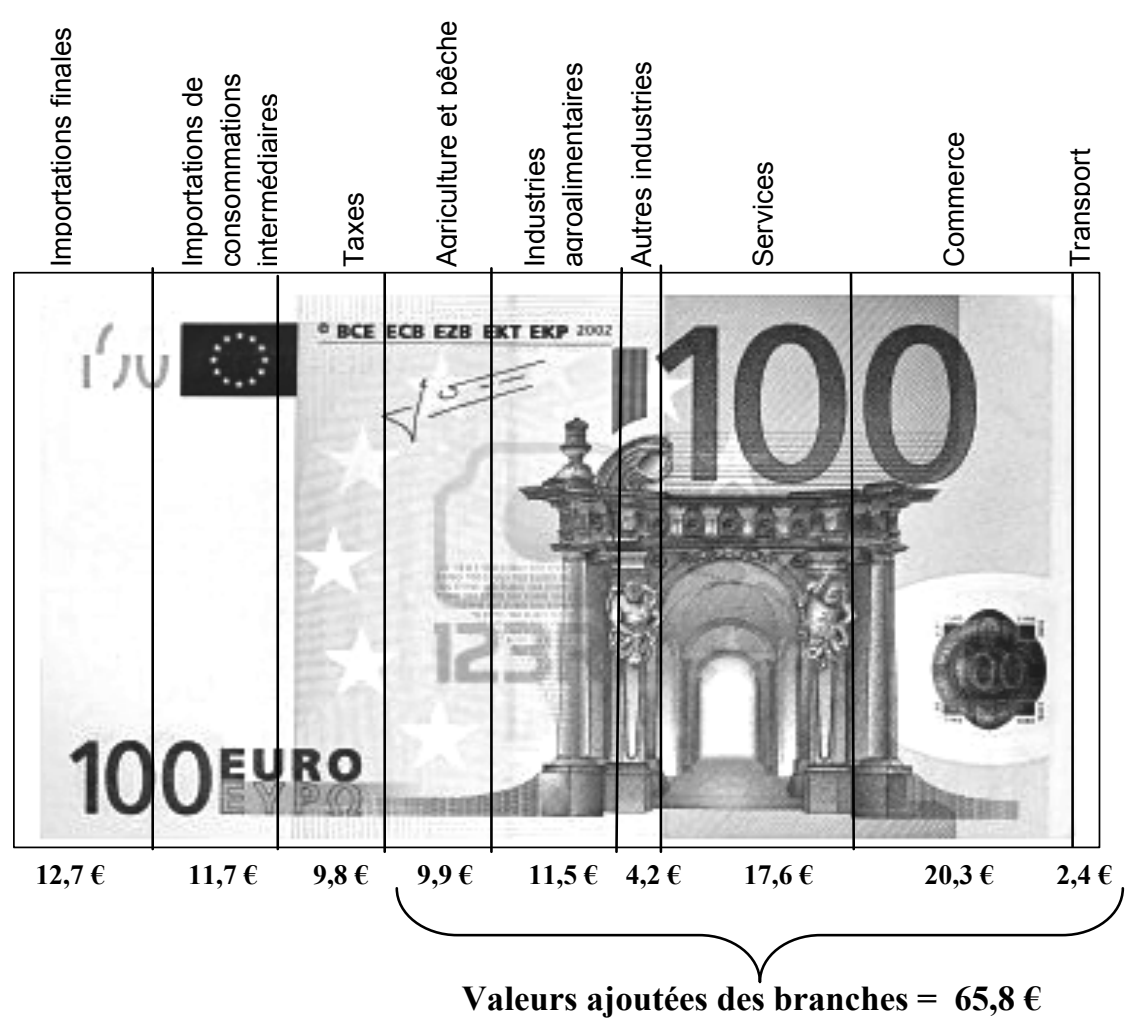

Sources : Eurostat, INSEE, calcul INRA.

de la consommation, des productions et des importations alimentaires, entre 1995 et 2007 (graphiques 2 et 3).

La consommation alimentaire crôit, en volume, à un rythme régulier de $1 \%$ par an. Le volume de la production agricole fluctue, avec une forte baisse en 2003 due à des aléas climatiques ayant réduit les récoltes céréales et de fruits, mais tend à stagner depuis 1998, ce qui est le cas également du volume de la production des IAA.

Les importations font un bond considérable, ce qui se traduit par une dégradation du rapport, en volume, entre exportations et importations, qui a baissé d'environ $15 \%$ entre 1995 et 2009 (Butault, Réquillart, 2012).

L'évolution des prix agricoles est déterminée par les réformes successives de la PAC d'une part, et la variation des cours mondiaux d'autre part. Ces réformes successives $(1992,1999,2003)$ se traduisent par un alignement des prix intérieurs sur les cours mondiaux dans un contexte de baisse de ceux-ci d'abord, puis dans un contexte de hausse avec un pic en 2007. Les prix agricoles à la production baissent donc en termes réels jusqu'en 2006, puis remontent. Avant l'application de la réforme de 2003 en 2006, instaurant le découplage des aides, la baisse 
Graphique 2. Évolution des volumes de la consommation, des productions et des importations alimentaires, de 1995 à 2007

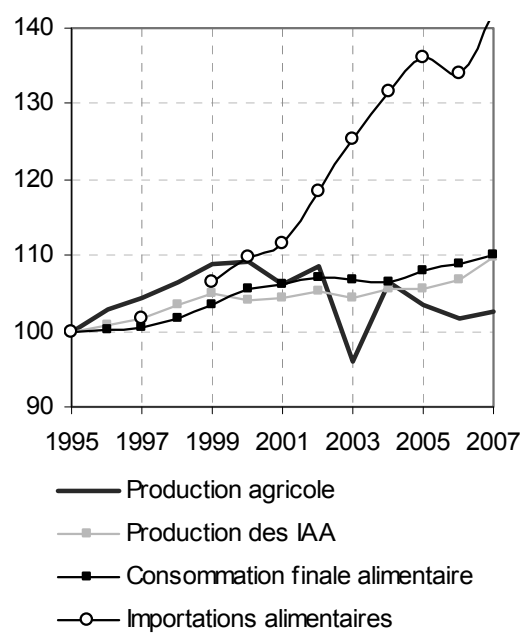

du prix de base est plus limitée que celle des prix à la production, compte tenu de l'augmentation des subventions liées aux produits.

Comme dans les années antérieures, mais peut-être pour des raisons différentes $^{5}$, la variation des prix agricoles se transmet relativement peu aux prix des produits des IAA, et ceux-ci restent relativement stables en termes réels. Le prix des importations en produits alimentaires baisse régulièrement.

Jusqu'en 2004, en termes réels, les prix à la consommation augmentent régulièrement et plus sensiblement que les prix agricoles et ceux des IAA, donc probablement sous l'effet d'une hausse des marges du commerce. Une nouvelle phase de hausse s'observe ensuite à partir de 2007

5. Les gains de productivité sont très faibles dans les IAA, ce qui explique le relatif maintien des prix à la production malgré la baisse des prix agricoles (Butault, 2008 ; Butault et al., 2012).
Graphique 3. Évolution des prix réels de la consommation, des productions et des importations alimentaires, de 1995 à 2007

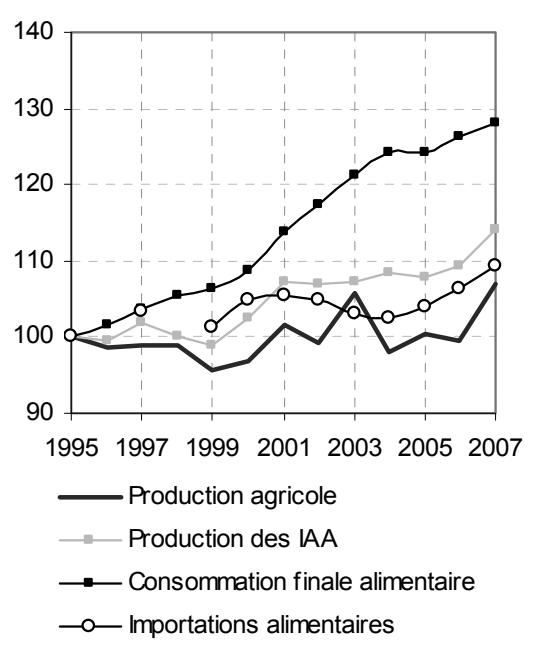

Source : Eurostat, INSEE, calcul INRA.

pour les prix à la consommation, en partie sous l'effet de celle des prix agricoles.

\section{Le contenu en importations \\ de la consommation alimentaire}

En apparence, le graphique 4 semble montrer une certaine stabilité de la décomposition de l'euro alimentaire. Un examen plus fin amène à des conclusions plus nuancées, en mettant en évidence des tendances certes lentes, mais lourdes dans l'évolution.

Une première tendance est l'augmentation du poids des importations dont la part totale (importations finales et en consommations intermédiaires) dans l'euro alimentaire passe de 22,6 à 26,1 \% (graphique 5). Cette augmentation concerne autant les produits alimentaires finals que les produits entrant dans le cycle de production.

Un point important est la part prépondérante des importations d'origine intracommunautaire, peu modifiée par l'élargissement de l'Union européenne : $65 \%$ en 2007 comme en 1995 (graphiques 6 et 7). 
RECHERCHES

Philippe BOYER, Jean-Pierre BUTAULT

Graphique 4. Décomposition de l'euro alimentaire de 1995 à 2007

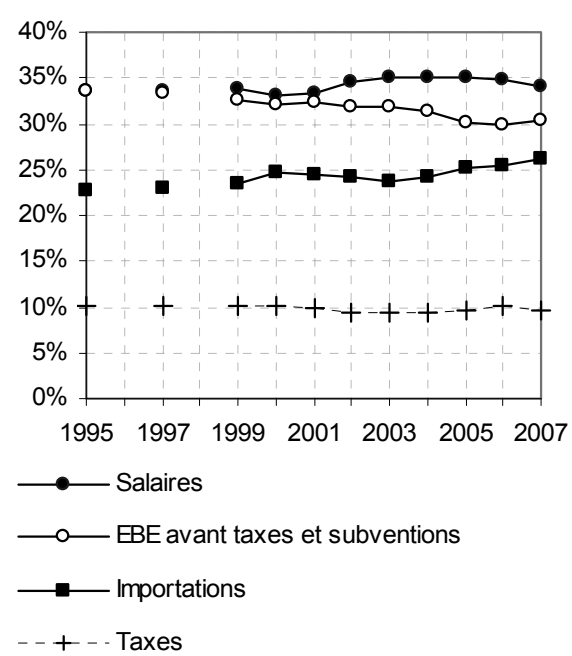

Graphique 6.Composition des importations directes et indirectes entrant en contenu de l'euro alimentaire en 1995

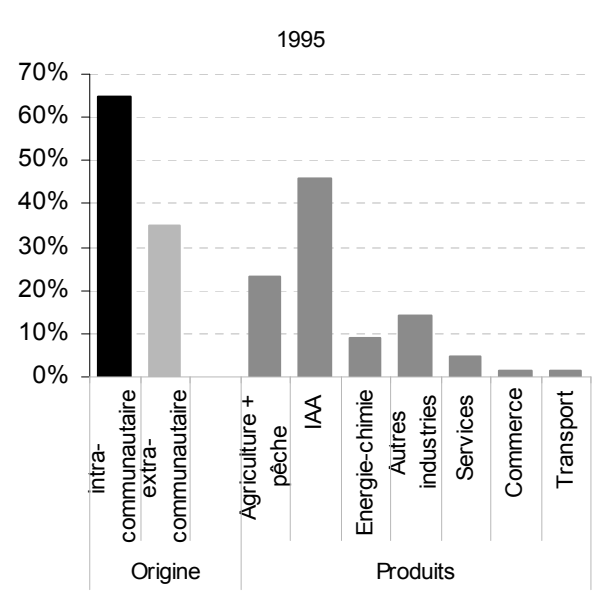

Le poids des importations tient ainsi pour une part au développement du commerce intracommunautaire qui peut porter sur des
Graphique 5. Évolution de la part des importations dans l'euro alimentaire de 1995 à 2007

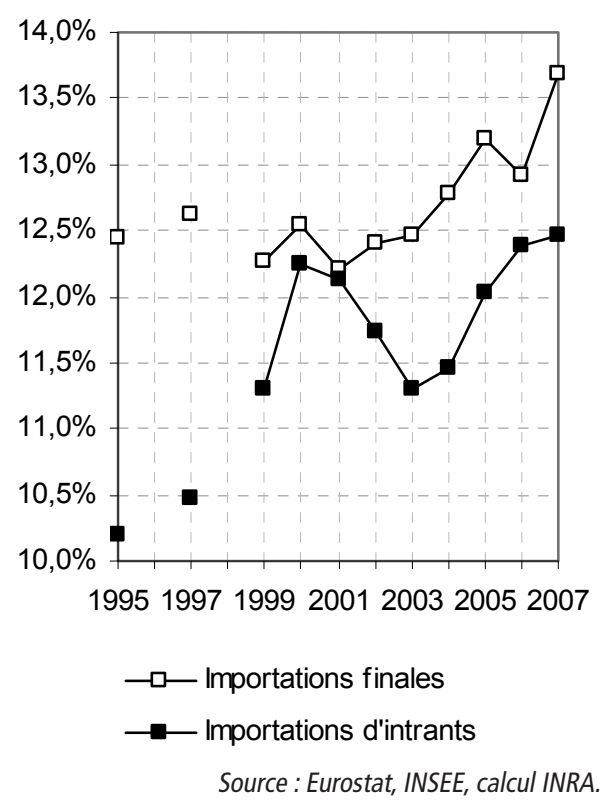

Graphique 7.Composition des importations directes et indirectes entrant en contenu de l'euro alimentaire en 2007

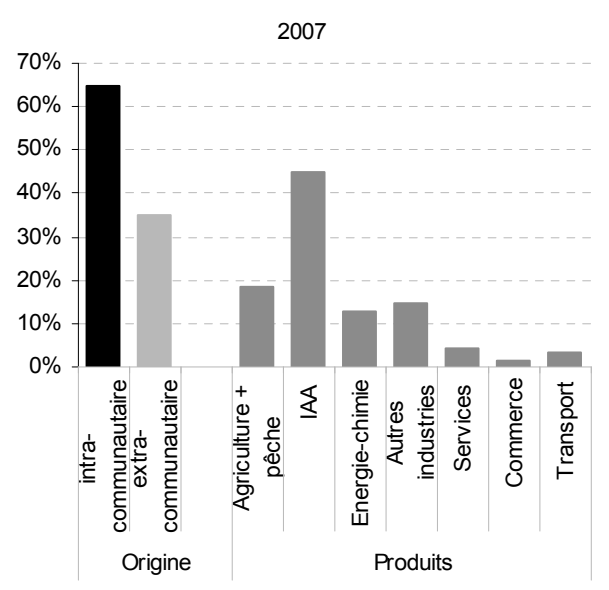

Source : Eurostat, INSEE, calcul INRA.

produits similaires. Il serait ainsi beaucoup plus faible si cet exercice de décomposition était mené sur l'Union européenne 
(des travaux en ce sens sont en cours), et non sur la France.

La part des importations en produits de l'agriculture et des IAA, constituée d'une demande finale ou de consommations intermédiaires, est bien entendu la plus importante, mais baisse entre 1995 (69\%) et 2007 (63\%). Cette baisse est compensée par une hausse de la part des produits industriels, notamment des branches de l'énergie et de la chimie, part qui est bien sûr très sensible aux variations de prix. Les importations en produits des branches des services, du commerce, ou même du transport sont d'un montant très faible.

\section{Le contenu en produits intérieurs} de la consommation domestique

Avant d'aborder le partage des valeurs ajoutées, on peut présenter, dans une

Graphique 8. Production intérieure en euros pour 100 euros (hors taxe) de consommation de produits d'origine domestique de 1995 à 1997

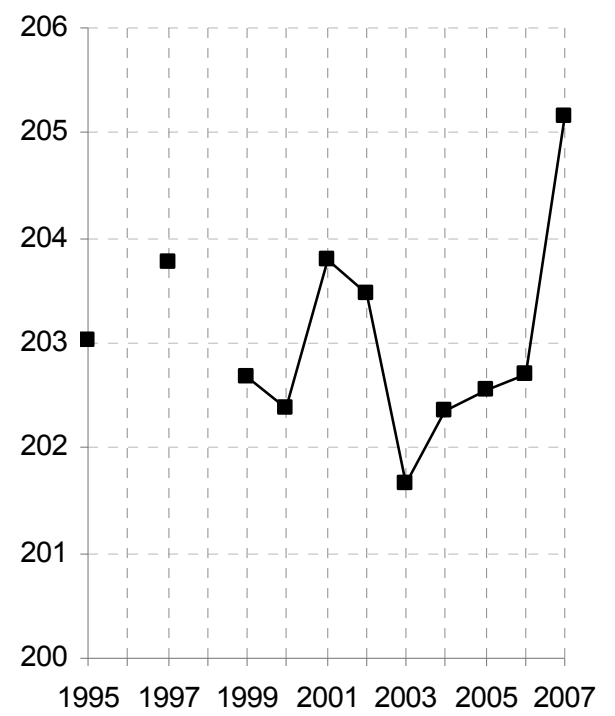

problématique Leontief, les coefficients de production. Ceux-ci sont donnés, dans le graphique 8 , pour la production intérieure par rapport à la consommation en produits d'origine domestique.

Le coefficient global de production sur la consommation reste relativement stable : pour 100 euros de consommation de produits domestiques, le montant de la production intérieure ne varie qu'entre 202 et 205 euros, de 1995 à 2007.

La structure de ce coefficient se modifie par contre sensiblement (graphique 9) :

- Le montant de la production agricole passe de 35 à 30 euros et celui des IAA de 74 à 66 euros.

- Le montant de la production du commerce gagne 5 euros et celui des services 10 euros.
Graphique 9. Production des branches pour 100 euros (hors taxe) de consommation de produits d'origine domestique de 1995 à 1997

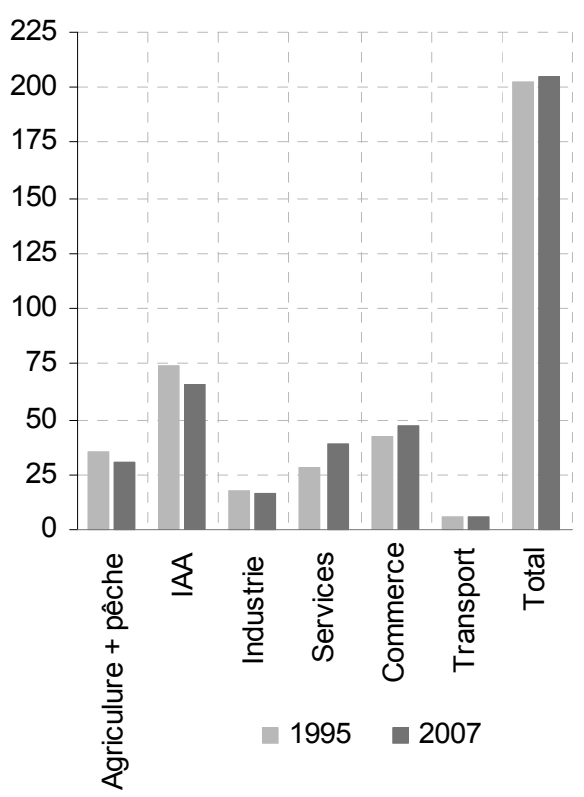

Source : Eurostat, INSEE, calcul INRA. 
RECHERCHES

On peut noter une régression de la part de l'industrie alors que la valeur des biens industriels importés progresse.

\section{Le partage des valeurs ajoutées}

Compte tenu de la progression des importations, les taxes étant fixes, la part de la valeur ajoutée des branches générée par la consommation alimentaire baisse et passe, entre 1995 et 2007, de 67 à $64 \%$ ( $\mathrm{gra}$ phique 10). Cette baisse affecte plus l'EBE des entreprises (avant taxe et subvention d'exploitation) que les salaires ${ }^{6}$.

Le partage de la valeur ajoutée entre branches ne dépend pas seulement des coefficients de production que nous venons d'analyser, mais aussi des taux de la

Graphique 10. Part des composants de la valeur ajoutée (salaires, EBE avant transferts) dans l'euro alimentaire entre 1995 et 2007, pour $100 €$

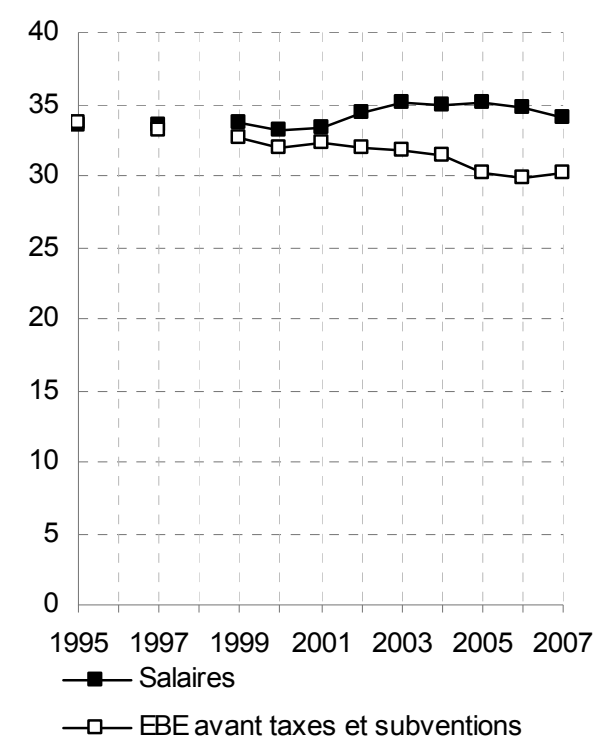

6. Le partage de la valeur ajoutée par branche entre salaires et EBE (avant taxes et subventions d'exploitation) dépend de la structure des entreprises, selon leur statut. Le texte se limite à analyser la valeur ajoutée. Le tableau en annexe donne le partage de cette valeur ajoutée. valeur ajoutée. En 2005, ce taux n'est que de $33 \%$ dans les IAA, $50 \%$ dans l'agriculture, mais il atteint $60 \%$ dans le commerce et $65 \%$ dans les services marchands.

L'observation des résultats moyens 1995-2007 montrait des parts relativement faibles des valeurs ajoutées de l'agriculture, de la pêche et des IAA dans l'euro alimentaire. Ces parts sont en outre en régression :

- La part de la valeur ajoutée agricole passe ainsi de 12 à $8 \%$ entre 1995 et 2005 (graphique 11). La remontée des prix agricoles en 2007 ne porte cette part qu'à un niveau légèrement supérieur à $9 \%$. Rappelons qu'il s'agit de la valeur ajoutée induite dans l'agriculture par la

Graphique 11. Part de la valeur ajoutée de l'agriculture, de la pêche et des IAA dans l'euro alimentaire entre 1995 et 2007 , pour $100 €$

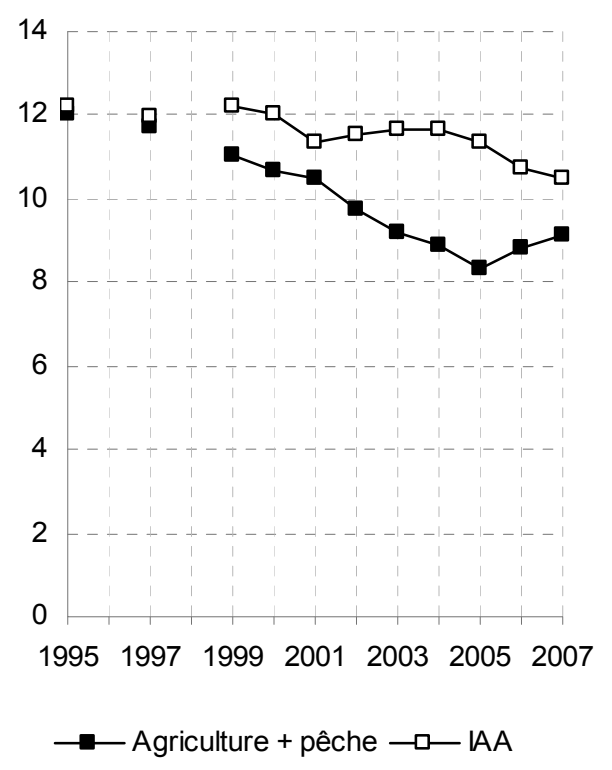

Source : Eurostat, INSEE, calcul INRA. 
Graphique 12. Part de la valeur ajoutée des services et du commerce dans l'euro alimentaire entre 1995 et 2007 , pour $100 €$

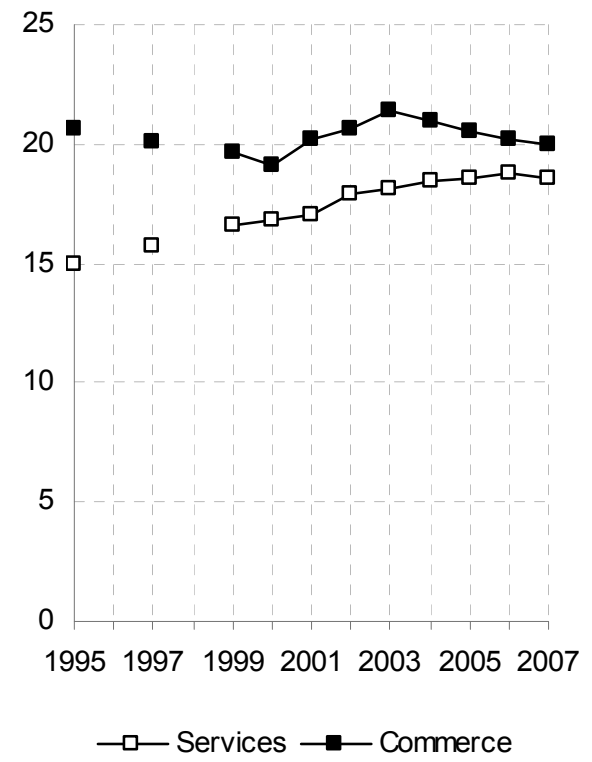

demande finale du consommateur, donc hors aides directes aux produits supportées par le contribuable?

- La régression de la part de la valeur ajoutée des IAA est quasiment linéaire. Cette part n'est plus que de $10,5 \%$ en 2007 contre $12 \%$ en 1995.

Les autres branches profitent donc de cette régression de l'agriculture et des IAA dans l'euro alimentaire :

7. En 2005, la valeur ajoutée de la branche agricole est, par exemple, de 23,4 milliards d'euros, mais de 31,6 milliards si on ajoute les subventions (8,1 milliards), soit $26 \%$. Comme on l'a dit en note 1, la méthode permet aussi de calculer la part des emplois finals dans la valeur ajoutée des branches. Sur les 23,4 milliards de valeur ajoutée de la branche agricole, 12,2 milliards incombent à la consommation alimentaire, 7,3 milliards étant issus des exportations et 4 milliards aux autres emplois (Butault, Boyer, 2012).
Graphique 13. Part de la valeur ajoutée des autres industries et du transport dans l'euro alimentaire entre 1995 et 2007 , pour $100 €$

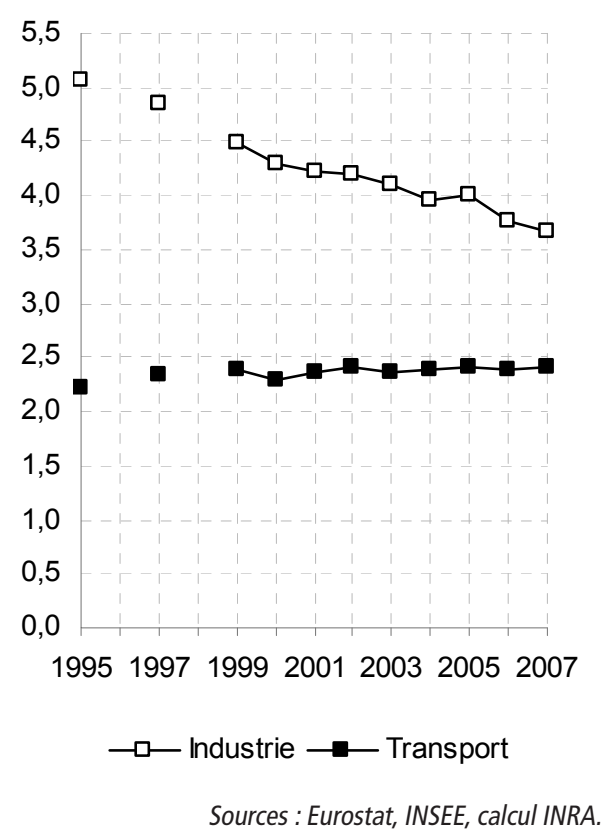

- La part de la valeur ajoutée des services (graphique 12) fait notamment un bond considérable en passant de $15 \%$ en 1995 à près de $19 \%$ en 2007.

- L'évolution de la part de la valeur ajoutée du commerce est plus irrégulière. Cette part perd un point et demi de 1995 à 2000, puis remonte pour s'établir à $21,4 \%$ en 2003 , de nombreux commentateurs ayant attribué cette hausse à l'effet de la loi Galland (Butault, 2008). Elle retombe en 2007 à $20 \%$, soit à un niveau presque équivalent à celui de 1995. Ceci renvoie au tassement des prix à la consommation en fin de période (graphique 2), malgré la hausse des prix agricoles.

La part de la valeur ajoutée des industries autres que les IAA régresse régulièrement et passe de 5,1 à 3,7\% entre 1995 et 2007. Cette baisse peut être mise en relation avec 
RECHERCHES

l'accroissement des importations, mais aussi avec l'augmentation du poids des services dont une part semble imputable, à travers les TES, au recours croissant des IAA à des entreprises de services industriels (intérim, informatique, publicité...). La part de la valeur ajoutée des transports reste stable, quant à elle, autour de $2 \%$.

\section{Le contenu en emplois}

L'emploi est cerné, dans les TES, par le nombre de personnes employées, critère qui n'est pas le plus pertinent dans la mesure où il ne prend pas en compte le temps de travail. Ce nombre d'emplois, induit par la consommation alimentaire, passe de 1853 à 1827 milliers entre 1995 et 2007, avec un pic de 1982 milliers en 2003.

L'emploi agricole perd 100000 personnes. La régression des industries autres

Graphique 14. Nombre de personnes employées pour la consommation alimentaire entre 1995 et 2007 (en milliers)

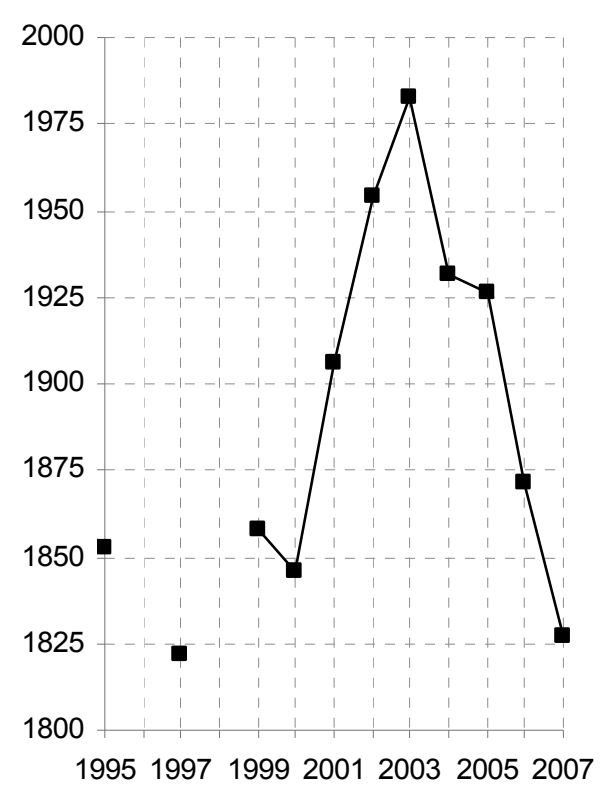

que les IAA dans la consommation alimentaire se confirme en termes d'emplois. Les IAA présentent, par contre, un emploi en légère progression malgré la baisse de leur part dans la valeur ajoutée. Les effectifs sont stables dans le transport. La consommation alimentaire induit, dans le commerce, 623000 emplois en 1995 et 662000 en 2007. Mais, c'est dans les services que le gain d'emplois est le plus important : +60 000 entre 1995 et 2007, avec 300000 personnes employées en 2007. La hausse de la contribution des services dans la valeur ajoutée générée par la consommation alimentaire n'est pas seulement due à un effet « prix » : elle correspond à un important développement en « volume » de ces activités.

Graphique 15. Nombre de personnes employées par branche pour la consommation alimentaire en 1995 et en 2007 (en milliers)

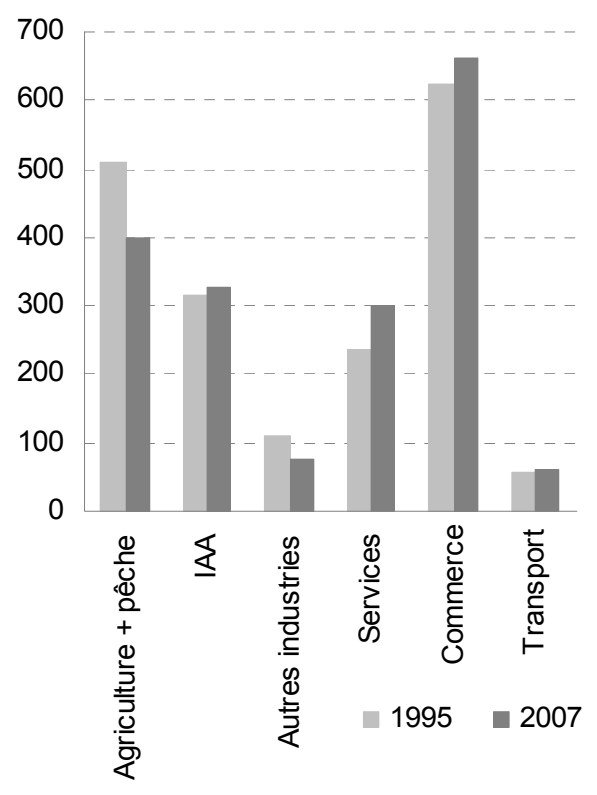

Sources: Eurostat, INSEE, calcul INRA. 
L'accroissement du poids des services Un retour aux données primaires

Avec la baisse de la part de valeur ajoutée agricole qui est explicable par des effets « prix », l'accroissement du poids des services est l'un des résultats les plus marquants de l'étude. Il est bien sûr plus fort sur la valeur ajoutée que sur la production, compte tenu du taux élevé de valeur ajoutée dans la branche des services. Plusieurs études récentes convergent sur cet accroissement du poids des services, notamment dans les IAA, et pour le comprendre, on peut revenir sur les données primaires. Le graphique 16 donne ainsi la part des services dans les consommations d'origine intérieure (au prix de base, avant correction sur le vin et les subventions agricoles) en 1995 et 2007, pour les branches de l'agriculture, des IAA et du commerce.

Graphique 16. Part des services dans les consommations intermédiaires d'origine intérieure en 1995 et 2007 (au prix de base)

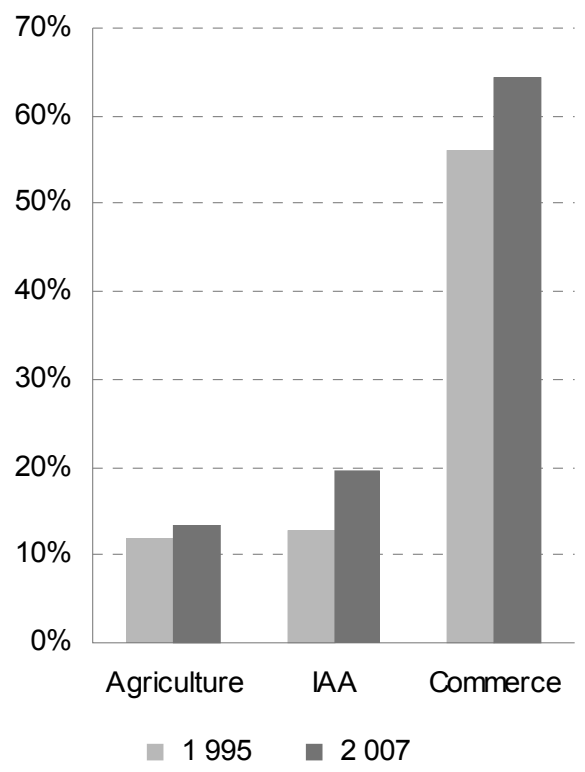

Sources : Eurostat.
L'accroissement de la part des services dans les consommations d'origine intérieure apparaît limité dans l'agriculture, cette part passant de 11,9 à 13,4\%. Elle est en fait mal prise en compte dans les TES, la branche agricole incluant l'activité des entreprises de travaux agricoles, laquelle s'est développée. Ces services apparaissent ainsi en production et en intraconsommation de la branche. De ce point de vue, l'accroissement du poids des services est sous-estimé pour l'agriculture, ce point marquant les limites des catégories statistiques.

Le poids des services est par contre très fortement croissant dans les consommations intermédiaires des IAA. Ceci confirme le résultat d'autres études ; Demmou (2010) montrant, par exemple, un recours accru des IAA à l'intérim et une externalisation de certaines tâches de l'industrie vers le secteur des services. Le phénomène de « désindustrialisation » peut ainsi être gonflé par les catégories statistiques. Le poids dans le chiffre d'affaires des IAA de l'ensemble des « autres achats et charges externes » progresse toutefois de plus de $30 \%$ entre 2000 et 2007 (Agreste, 2010). Ce poste recouvre, en partie, des achats auprès de branches classées dans les services dans les TES, et notamment des dépenses en publicité et communication facturées par la grande distribution dans le cadre de la coopération commerciale ou bien des " marges arrières » (Bastélica, Doisneau, 2004), en augmentation sur la période. Les « charges externes » des IAA englobent également que le recours à des sociétés de services informatiques et de conseil pour satisfaire les exigences sanitaires de la « traçabilité » également croissantes sur la période.

Le poids des services s'accroît également dans le commerce, ce qui, compte tenu de la méthode, se reporte sur la consommation alimentaire. 
RECHERCHES

**

Avant de conclure, il convient de souligner à nouveau les limites inhérentes de ce travail à toutes les utilisations des TES. Toutes les décompositions sont effectuées dans le cadre d'hypothèses de type Leontief : les coefficients de production et la structure de la valeur ajoutée des branches (y compris pour le commerce et le transport, dans le traitement des marges commerciales) sont fixes, quelle que soit l'utilisation des produits en consommations intermédiaires ou en demandes finales, en consommations finales ou en exportations. Cette hypothèse est très réductrice dans la mesure où, pour ne prendre qu' un exemple, pour une branche donnée, on ne consomme pas et on n'exporte pas les mêmes produits, du moins dans la même proportion ${ }^{8}$.

Les résultats font toutefois apparaître des tendances assez lourdes pour qu'on puisse en tirer des enseignements généraux. Ils permettent d'alimenter, de façon complémentaire à d'autres études, le débat actuel sur la formation des prix alimentaires.

Une part de ces tendances lourdes reflète des évolutions qui ne sont pas spécifiques à la consommation alimentaire et qui font, de manière générale, l'objet de débats en France. Ces tendances sont l'augmentation des importations, la baisse des emplois intérieurs dans les secteurs primaire et

8. Ainsi, dans le cadre « Leontief », la structure des intrants des produits de consommation finale est la même que celle des exportations, d'où certains biais : le taux élevé de valeur ajoutée sur les boissons, largement exportées, majore probablement le calcul de la valeur ajoutée induite par la consommation domestique dans les IAA. À l'inverse, les exportations agroalimentaires comprennent des matières premières non transformées, d'où cette fois une sous-estimation de la valeur ajoutée de l'agriculture et des IAA dans la consommation. secondaire, l'augmentation de la part des services... On retrouve les termes des débats sur la délocalisation des productions, la désindustrialisation de la France...

L'une des évolutions majeures reste, du moins jusqu'en 2005, la diminution de la part de la valeur ajoutée agricole dans la consommation alimentaire. Avec les réformes de 1992 et 1999, les prix agricoles intérieurs continuent à baisser dans la mesure où ils sont alignés sur les cours mondiaux, eux-mêmes aussi en diminution. Ce contexte devrait changer d'une part parce que ce processus est aujourd'hui presque achevé et d'autre part parce que les prévisions sur l'évolution des cours mondiaux (du moins en ce qui concerne les produits végétaux) sont plutôt à la hausse, mais avec une accentuation de leur variabilité. On peut douter toutefois que ces évolutions récentes se traduisent par une inversion forte de la tendance à la réduction de la part de la valeur ajoutée agricole : le contexte de 2007, avec un niveau élevé du prix des produits de grandes cultures, ne s'est traduit que par une faible augmentation de la part de la valeur ajoutée de l'agriculture dans la consommation alimentaire. Pour maintenir un certain niveau et une stabilité aux revenus des producteurs agricoles, la loi de modernisation de l'agriculture de 2010 a mis en place un système de contractualisation entre les producteurs et les premiers acheteurs. Pour être efficaces, ces contrats supposent que ces premiers acheteurs puissent contrôler le marché, ce qui renvoie à la question de la situation des IAA.

La part de la valeur ajoutée des IAA dans la consommation alimentaire se réduit, en effet, faiblement, mais de façon régulière, et ce, malgré une progression des emplois. Cette observation confirme le constat d'une fragilité globale des IAA, pointée dans de nombreuses études, renvoyant toutefois à des situations très contrastées selon les firmes. Dans la période récente, les gains de productivité des 
IAA sont quasiment nuls (Bontemps et al., 2010), et le secteur semble apparaître plutôt dans un rapport défavorable par rapport à la grande distribution, pour compenser cette faiblesse des gains de productivité, ce qui se traduit par des taux de rentabilité assez bas (Chantrel et al., 2009). Les difficultés rencontrées par certaines firmes du secteur dans la période récente attestent de cette situation. On peut ainsi douter de l'efficacité du système de contractualisation si les premiers acheteurs n'ont aucun pouvoir de marché par rapport à la grande distribution.

L'une des tendances les plus nettes est enfin l'augmentation de la part de la valeur ajoutée des services dans la consommation alimentaire (et ce alors même que l'analyse n'a pu prendre en compte la consommation via la restauration sur place ou à emporter). Ceci traduit une transformation de fond des modes de production, de distribution et de consommation des produits alimentaires. Les catégories statistiques utilisées sont sans doute insuffisantes pour rendre compte de ces transformations. Des analyses plus fines, tant sur le plan économique que sur le plan sociologique, sont nécessaires pour cerner ces évolutions. L'expertise récente de l'INRA (Esnouf et al., 2011) donne une synthèse sur l'évolution des systèmes alimentaires, en revoyant à la question de leur durabilité.

Du point de vue de cette durabilité, la baisse de la contribution de l'agriculture et de sa valeur ajoutée dans la consommation alimentaire peut poser problème dans la mesure où l'agriculture n'est pas seulement un secteur produisant des marchandises, mais joue un rôle important dans l'occupation du territoire. Une solution est de faire payer le consommateur pour la première fonction et le contribuable pour la seconde. D'un point de vue social, elle n'est pas nécessairement optimale, d'où l'idée d'attacher les productions agricoles au territoire, à travers la production de proximité, les labels... Il s'agit en fait, pour les agriculteurs, de capter une partie des services et des marges incluse dans la consommation alimentaire? ${ }^{9}$ Mais, la grande distribution s'emploie aussi à assurer ces fonctions.
9. Évolutions qui ne seraient pas nécessairement captées dans les TES ! 


\section{RÉFÉRENCES BIBLIOGRAPHIQUES}

Agreste (2010). Enquête annuelle d'entreprises. Comptes de résultats et soldes intermédiaires. Séries 1998-2006, résultats 2007. Service de la statistique et de la prospective, Ministère de l'Agriculture, de l'Agroalimentaire et de la Forêt, www.agreste.gouv.fr.

Allain M.-L., Chambolle C. (2003). Les relations entre la grande distribution et ses fournisseurs. Bilan et limites de trente ans de régulation. Revue Française d'Économie, vol. XVII, n 4, p. 169-212.

Bastélica C., Doisneau L. (2004). La publicité des industries agroalimentaires : les grandes entreprises s'affichent. Agreste Primeur, $\mathrm{n}^{\circ} 152$, novembre, Service de la Statistique et de la Prospective, Ministère de l'Agriculture, de l'Agroalimentaire et de la Forêt, 4 p.

Bontemps C., Maigné É., Réquillart V. (2010). La productivité de l'agro-alimentaire français de 1996 à 2006. Working paper TSE $\mathrm{n}^{\circ} 10-143$. À paraître dans Économie et Prévision.

Braibant M. (2008). La synthèse d'un tableau entrées-sorties en année courante, base 2000. Paris, INSEE, 110 p.

Braibant M. (2011). La confection d'un TES symétrique pour Eurostat et d'un tableau de contenu en importation. Paris, INSEE, $55 \mathrm{p}$.

Besson E. (2008). Formation des prix alimentaires. Rapport au Premier ministre, $58 \mathrm{p}$.

Butault J.-P., Boyer P. (2012). La décomposition de "l'euro alimentaire » en revenus des différents facteurs en France en 2005. Document de travail INRA-FranceAgriMerOFPMA, 26 p.

Butault J.-P., Réquillart V. (2012). L'agriculture et l'agro-alimentaire français à la recherche d'une compétitivité perdue. INRA Sciences Sociales, vol. 4-5/11, 4 p.

Butault J.-P. (2004). Les soutiens à l'agriculture : théorie, histoire, mesure. Paris, Les éditions de l'INRA, coll. « Mieux comprendre », $309 \mathrm{p}$.

Butault J.-P. (2007). La réforme de la PAC de 2003 : ère nouvelle ou fin de la PAC. Références INSEE, p. 152-165.
Butault J.-P. (2008). La relation entre prix agricoles et alimentaires. Revue Française d'Économie, vol. ${ }^{\circ} \mathrm{XXIII}, \mathrm{n}^{\circ} 2$, p. 215-241.

Canivet G. (2004). Restaurer la concurrence par les prix. Les produits de grande consommation et les relations entre industrie et commerce. Rapport au Ministre des Finances, Paris, La Documentation Française, 164 p.

Canning P. (2011). A revised and expanded food dollar series. A better understanding of food costs. Economics Research, Report $\mathrm{n}^{\circ} 114$, USDA, Washington DC, $42 \mathrm{p}$.

Chambolle C., Muniesa L., Ravo M. A. (2007). Concentration et puissance d'achat. Économie et Prévision, $\mathrm{n}^{\circ}$ 178-179.

Chantrel E., Lecocq P. E (2009). Les marges dans la filière agro-alimentaire en France. Trésor-Eco, $\mathrm{n}^{\circ} 53,8 \mathrm{p}$.

Cotis J.-P. (2009). Partage de la valeur ajoutée, partage des profits et écarts de rémunérations en France. Rapport au Président de la République, INSEE, mai, 90 p.

Demmou L. (2010). La désindustrialisation en France. Cahiers, Documents de travail de la DG Trésor, $\mathrm{n}^{\circ} 1$, juin, $52 \mathrm{p}$.

Esnouf C., Russel M., Bricat N. (2011). Pour une alimentation durable. Réflexions stratégiques duALIne, Paris, Éditions Quae, 288 p.

Eurostat (2008). Manual of supply, use and input-output tables. Luxembourg, 592 p.

Hassan D., Simioni M. (2004). Transmission des prix dans la filière des fruits et légumes : une application des tests de cointégration avec seuils. Économie rurale, vol. 283, n ${ }^{\circ} 1$, p. 27-46.

Lambert C. (2009). Les modalités de formation des prix alimentaires : du producteur au consommateur. Rapport du Conseil économique, social et environnemental, $170 \mathrm{p}$.

Leontief W. (1966, 1986). Input-output Economics. New York, Oxford University Press, $1^{\text {st }}$ and $2^{\text {nd }}$ ed., $257 \mathrm{p}$.

Observatoire de la formation des prix et des marges des produits alimentaires (2012). Prix et coûts dans l'agroalimentaire. Nouvelles études : comptes par rayon des 
GMS, l'euro alimentaire. Rapport au parlement, FranceAgriMer, MAAF, MEF, octobre, $411 \mathrm{p}$.

Rastoin J.-L., Ghersi G. (2010). Le système alimentaire mondial. Concepts et méthodes, analyses et dynamiques. Paris, Éditions Quæ, coll. « Synthèse », 565 p.

Réquillart V., Simioni M., Xosé-Luis V. I. (2008). Imperfect competition in the fresh tomato industry. Communication au $12^{\text {th }}$ Congres of the European Association of Agricultural Economics, Gand Août 2008, Gremaq-Inra Toulouse, $11 \mathrm{p}$.
Rouchet J. (2002). Évolution des prix agricoles et alimentaires. Document de travail INSEE 2002/08, 146 p.

Schluter G., Lee C., Leblanc M. (1998). The weakening relationships between farm and food prices. American Journal of Agricultural Economics, vol. 80, $\mathrm{n}^{\circ}$ 5, p. 1134-1138.

Stoléru L. (1967). L'équilibre et la croissance économique. Paris, Dunod, coll. «Finance et économie appliquée », 498 p. 
RECHERCHES

Philippe BOYER, Jean-Pierre BUTAULT

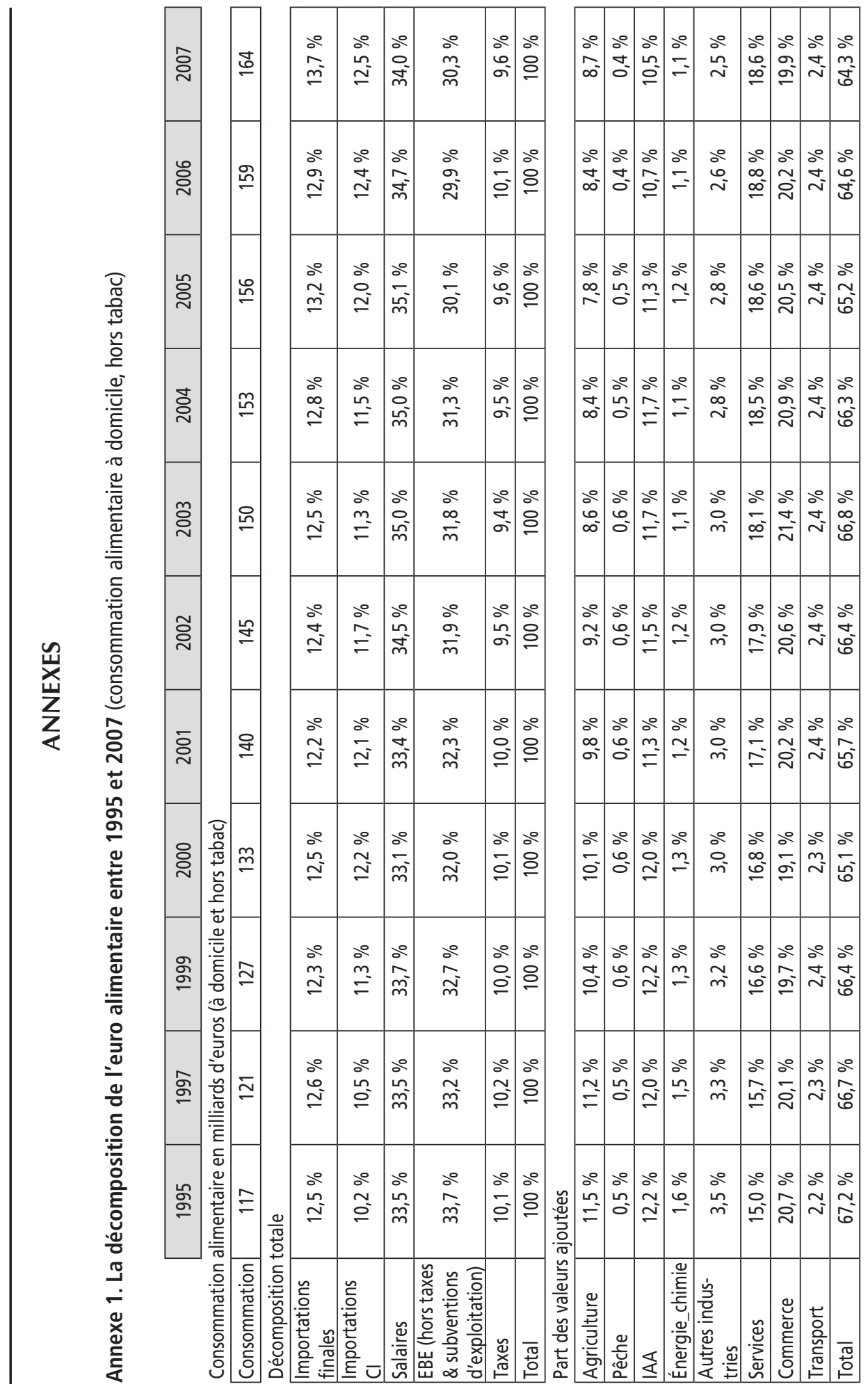


L'euro alimentaire et le partage des valeurs ajoutées

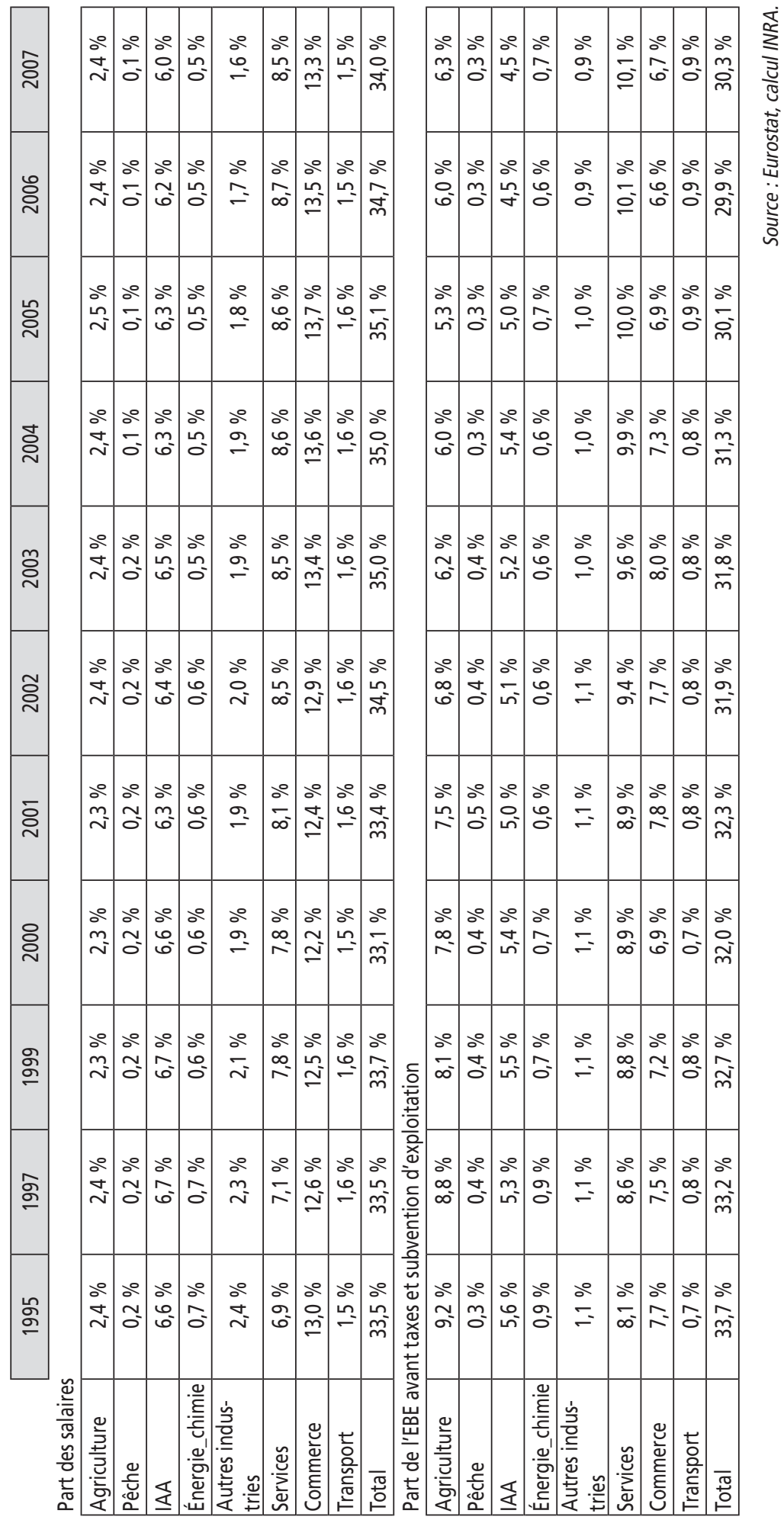

66 • Économie Rurale 342/Juillet-Août 2014 
RECHERCHES

Philippe BOYER, Jean-Pierre BUTAULT

\begin{tabular}{|c|c|c|c|c|c|c|c|c|c|c|c|c|}
\hline $\begin{array}{l}\text { बूँّ } \\
\stackrel{0}{\circ}\end{array}$ & 要 & & 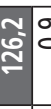 & & 咅 & $\begin{array}{l}\text { o̊ } \\
\text { 足 }\end{array}$ & $\begin{array}{l}\tilde{\pi} \\
\tilde{n}\end{array}$ & \begin{tabular}{|c|}
$m$ \\
$\overline{\tilde{m}}$ \\
\end{tabular} & $\begin{array}{l}0 \\
0 \\
0\end{array}$ & 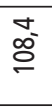 & 竝 & $\frac{\hat{m}}{\stackrel{m}{m}}$ \\
\hline 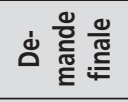 & $\overline{-}$ & $=$ & 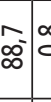 & s. & $\stackrel{\stackrel{n}{\Sigma}}{\equiv}$ & $\frac{m}{\frac{m}{q}}$ & ষ্ণ & 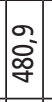 & 号 & $\bar{g}$ & 艿 & $\begin{array}{l}\text { ळ్- } \\
\text { ले } \\
\text { - }\end{array}$ \\
\hline 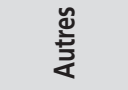 & $\bar{\sim}$ & 응 & $\hat{o}$ & 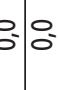 & $\mid$\begin{tabular}{l}
$n$ \\
\hdashline \\
$c$
\end{tabular} & @ి & $\begin{array}{l}\infty \\
\stackrel{2}{2}\end{array}$ & $\stackrel{\infty}{\sim}$ & $\stackrel{\searrow}{\infty}$ & $\bar{\sigma}$ & $\stackrel{\infty}{=}$ & 怘 \\
\hline $\begin{array}{l}\frac{t}{0} \\
\text { 훈 }\end{array}$ & $\approx$ & 응 & $\stackrel{\mathbb{N}}{\mathbf{N}}$ & s. & $\frac{n}{6}$ & ळે & $\begin{array}{l}\stackrel{6}{\circ} \\
\stackrel{m}{0}\end{array}$ & $\hat{\sim}$ & $\stackrel{\infty}{\infty}$ & $\underset{\simeq}{\cong}$ & $\bar{m}$ & ڤે \\
\hline 㝘 & $\begin{array}{l}m \\
\infty\end{array}$ & $\hat{\circ}$ & $\begin{array}{l}0 \\
\text { bे }\end{array}$ & & $\begin{array}{l}n \\
0 \\
0 \\
n\end{array}$ & 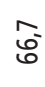 & हิ' & 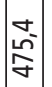 & $\begin{array}{l}0 \\
0_{0}^{\circ}\end{array}$ & $\bar{\pi}$ & ন̀ & $\begin{array}{l}\stackrel{\text { Ln }}{\circ} \\
\stackrel{0}{=}\end{array}$ \\
\hline
\end{tabular}

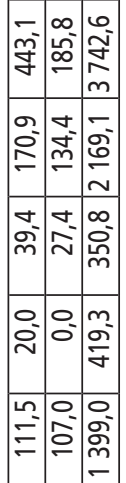

\begin{tabular}{|c|c|c|c|c|c|c|c|c|c|c|c|c|c|}
\hline సٓ & - & $\stackrel{-1}{-}$ & $\begin{array}{l}\stackrel{n}{2} \\
\stackrel{m}{m}\end{array}$ & ㅇ. & 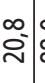 & & $\begin{array}{c}\text { ñ } \\
\grave{N}\end{array}$ & ָ̃ & ôn & 옹 & $\stackrel{\dot{m}}{\stackrel{\sim}{*}}$ & 兽 & $\stackrel{\infty}{\text { g- }}$ \\
\hline 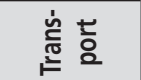 & 응 & 응 & $\tilde{\sigma}$ & 응. & $\cong$ & ๙ุ' & $\stackrel{\infty}{\leftarrow}$ & 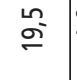 & $\stackrel{2}{-}$ & $\hat{\sim}$ & $\stackrel{\infty}{\circ}$ & 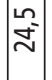 & $\frac{m}{6}$ \\
\hline 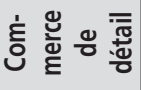 & 웅 & 웅 & : & ㅇ. & $\stackrel{\infty}{-}$ & $\stackrel{i}{N}$ & $\stackrel{\overbrace{}}{\sim}$ & $\stackrel{\infty}{\sim^{\sim}}$ & $=$ & $\stackrel{\infty}{\circ}$ & $\tilde{m}$ & $\left|\begin{array}{c}\infty \\
m\end{array}\right|$ & $\bar{m}$ \\
\hline 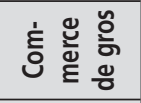 & $\because$ & 웅 & 灾 & ㅇ. & $\stackrel{\infty}{\sim}$ & $\stackrel{n}{m}$ & 늠 & $\stackrel{\infty}{\mathbb{f}}$ & $\stackrel{2}{-}$ & ్ㅏ & $\hat{o}$ & 으. & $\stackrel{\llcorner}{\stackrel{L}{0}^{\circ}}$ \\
\hline 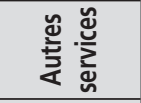 & 灾 & $\overline{0}$ & $\stackrel{\sim}{\approx}$ & ○. & $\stackrel{\infty}{\infty}$ & $\hat{\sigma}$ & $\stackrel{\infty}{\sim}$ & $\begin{array}{l}\circ \\
\text { g. }\end{array}$ & 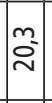 & $\stackrel{\circ}{\&}$ & $\bar{m}$ & $\left|\begin{array}{l}0 \\
\sigma^{-}\end{array}\right|$ & 吞 \\
\hline 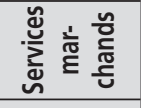 & 잉 & 웅 & $=$ & ○. & $\bar{\sigma}$ & $\stackrel{+}{\sigma}$ & $\stackrel{m}{m}$ & $\stackrel{\circ}{\stackrel{0}{J}}$ & $\underline{0}$ & $\begin{array}{l}\infty \\
\infty \\
\infty\end{array}$ & $\stackrel{\infty}{\sim}$ & $\left|\begin{array}{l}n \\
\infty \\
\infty\end{array}\right|$ & $\begin{array}{l}\stackrel{0}{0} \\
\stackrel{\sim}{m}\end{array}$ \\
\hline 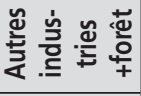 & $\bar{\sim}$ & 영 & $\infty$ & ㅇ. & $\bar{\sim}:$ & $\stackrel{0}{=}$ & 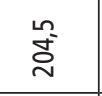 & ผั & $\begin{array}{l}\infty \\
0 \\
0\end{array}$ & $\stackrel{\text { ڤે }}{\sim}$ & $\stackrel{\stackrel{\llcorner}{\ddagger}}{ \pm}$ & क्ञ & $\bar{\infty}$ \\
\hline 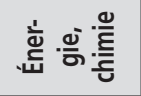 & $\approx$ & 잉 & $\stackrel{m}{=}$ & $\therefore$ & 0 & $\begin{array}{l}\infty \\
\text { m. }\end{array}$ & $\stackrel{\stackrel{n}{=}}{=}$ & $\stackrel{\bullet}{ \pm}$ & $\approx$ & F & $\stackrel{\overbrace{}}{\sim}$ & 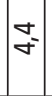 & $\underset{\infty}{\infty}$ \\
\hline 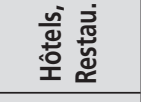 & $\cong$ & ma & Ln. & 웅 & i: & $\stackrel{n}{=}$ & $\stackrel{2}{\simeq}$ & न̃ & $\Xi$ & $\stackrel{\Xi}{\sim}$ & 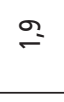 & $\bar{N}$ & $\underset{m}{\stackrel{m}{m}}$ \\
\hline $\begin{array}{l}\frac{\pi}{\pi} \\
\frac{\pi}{\sigma 0} \\
\frac{\pi}{\pi}\end{array}$ & $\bar{\sigma}$ & 영 & : & : & : & 응 & 웅 & $\bar{\sigma}$ & ㅇ. & 웅 & 응 & 응 & $\stackrel{m}{o}$ \\
\hline 委 & $\frac{0}{m}$ & 0 & $\begin{array}{l}\sim \\
\underline{n} \\
\end{array}$ & $\therefore$ & ठ্) & $\stackrel{n}{\sim}$ & छే & $\stackrel{\text { nn }}{n}$ & 8 & $\bar{E}$ & $\stackrel{\bullet}{m}$ & 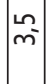 & స్ \\
\hline 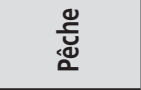 & 웅 & 웅 & 잉 & ㅇ. & 영 & $\tilde{c}$ & 웅 & $\bar{\sigma}$ & 응 & 응 & 응 & 응 & $\stackrel{n}{\alpha}$ \\
\hline 㝘章 & 它 & 웅 & $\underset{\nabla}{*}$ & : & $\begin{array}{l}0 \\
0\end{array}$ & 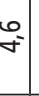 & $\tilde{m}$ & $\stackrel{\infty}{\sim}$ & 志 & $\stackrel{\circ}{i}$ & $\cong$ & శ్ & 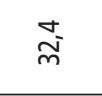 \\
\hline & \begin{tabular}{|l|} 
\\
$\frac{0}{3}$ \\
$\frac{3}{3}$ \\
$\frac{3}{0}$ \\
\end{tabular} & & 14 & 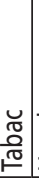 & $\bar{\Phi}$. & . & 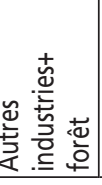 & 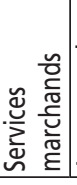 & 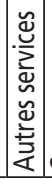 & 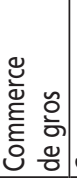 & 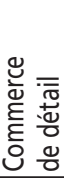 & 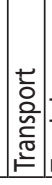 & 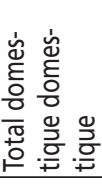 \\
\hline
\end{tabular}

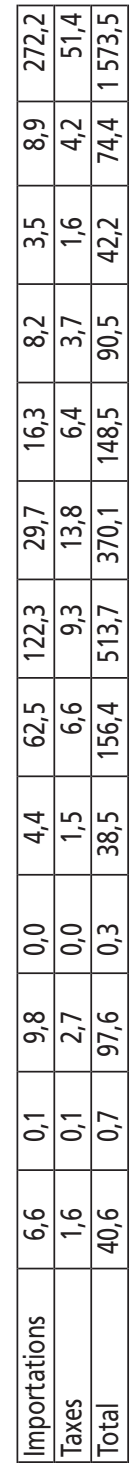


L'euro alimentaire et le partage des valeurs ajoutées

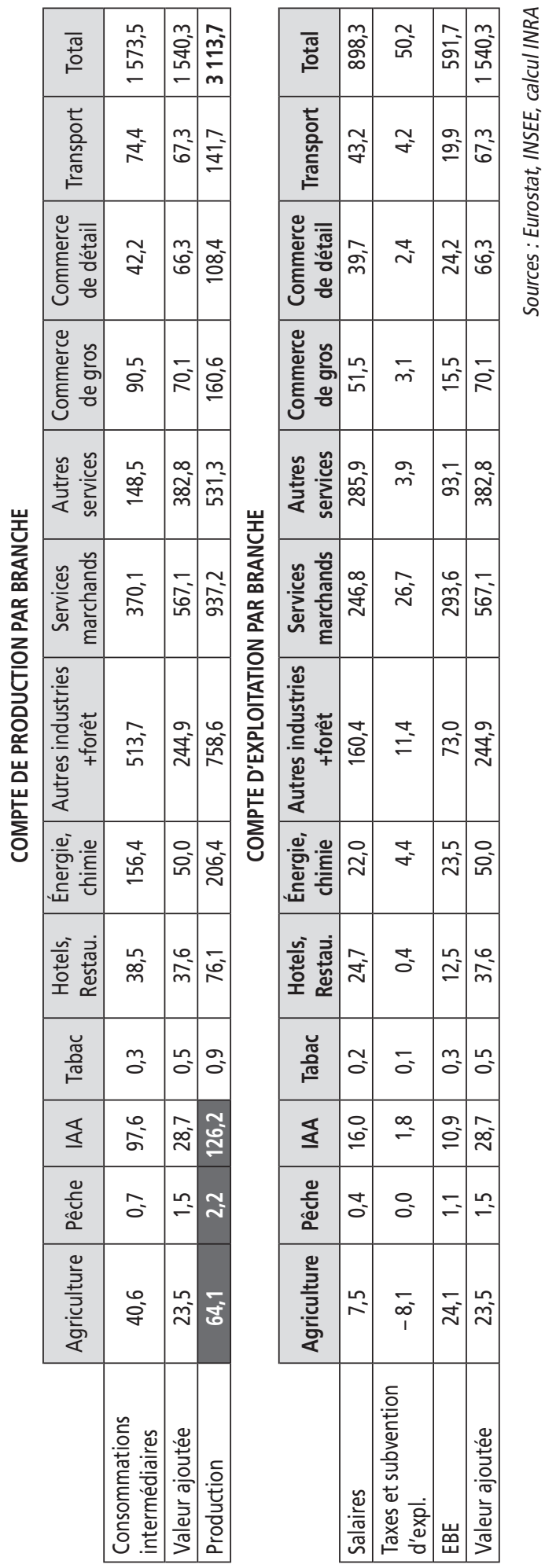

68 • Économie Rurale 342/Juillet-Août 2014 